JOURNAL OF

SYMPLECTIC GEOMETRY

Volume 1, Number 2, 171-196, 2002

\title{
Holomorphic discs and the complex Monge-Ampère equation
}

\author{
S.K. DONALDSON
}

\section{Introduction.}

In this paper we study a version of the Dirichlet problem for the homogeneous complex Monge-Ampère equation. Let $\left(V, \omega_{0}\right)$ be a compact Kahler manifold, of complex dimension $n$. We write $\Omega_{0}$ for the lift of $\omega_{0}$ to the product $D \times V$, where $D$ is the closed unit disc in $\mathbf{C}$, with boundary $\partial D=S^{1}$. If $T$ is a subset of $D$, a real-valued function $\Phi$ on $T \times V$ may be considered as a family of functions $\phi_{\tau}$ on $V$, parametrised by $\tau \in T$, with $\phi_{\tau}(x)=\Phi(\tau, x)$. We say that $\Omega_{0}+i \bar{\partial} \partial \Phi$ is positive on the vertical slices over $T$ if for each $\tau \in T$ the $(1,1)$-form $\omega_{0}+i \bar{\partial} \partial \phi_{\tau}$ on $V$ is strictly positive, in the usual sense. We will study the following

Problem 1. Suppose given a smooth function $F: V \times \partial D \rightarrow \mathbf{R}$ such that $\Omega_{0}+i \bar{\partial} \partial F$ is positive on the vertical slices over $\partial D$. Find a smooth function $\Phi: V \times D \rightarrow \mathbf{R}$ which agrees with $F$ on the boundary; with $\Omega_{0}+i \bar{\partial} \partial \Phi$ positive on all vertical slices over $D$ and which satisfies the Monge-Ampère equation

$$
\left(\Omega_{0}+i \bar{\partial} \partial \Phi\right)^{n+1}=0
$$

throughout $V \times D$.

This paper is one of a sequence [4], [5], [6] investigating connections between Kahler manifolds and symplectic geometry (a point of view which goes back to Mabuchi [8]). The boundary value problem above was introduced and discussed in [5]. It is shown there that the solution $\Phi$ to the problem, if it exists, is unique. Moreover it is also explained that the problem can be viewed as the search for an analogue of a standard factorisation theorem for loops in Lie groups, where the pertinent group in this case is the symplectomorphism group of $\left(V, \omega_{0}\right)$. More generally, one can pose the corresponding question where the disc is replaced by any Riemann surface-with-boundary and, as explained in [5], in the case of the cylinder with rotation-invariant 
data the problem can be viewed as the search for geodesics in the space of Kahler metrics. X-X. Chen has shown [2] that, with a suitable interpretation of the partial differential equation, there are always $C^{1,1}$ solutions to these boundary value problems for any smooth boundary data. Thus the issue remaining after Chen's work is the smoothness of the solution. The main question we address in this paper is the problem of deforming a smooth solution under a small change of the boundary data. Our first result is

Theorem 1. The set of smooth functions $F$ for which a smooth solution to Problem 1 exists is open in $C^{\infty}(V \times \partial D)$.

In fact our proof will show that this set is open in the $C^{2}$ topology. The set is obviously non-empty: for example the function $F=0$ admits the solution $\Phi=0$.

It seems quite likely that this result could be proved by applying the Nash-Moser implicit function theorem, following the approach of Moriyon [9] in a related problem. However in this paper we will take a different route and obtain Theorem 1 as a consequence of an alternative geometric interpretation of the problem, involving families of holomorphic discs. It is well-known that a solution $\Phi$ of the Monge-Ampère equation as in Problem 1 yields a family of holomorphic discs in $V$. The new observation in this paper is that these have natural holomorphic lifts to a larger space $\mathcal{W}_{V}$ (which can be identified locally with the cotangent bundle of $V$ ) and the solution can be reconstructed from these lifted discs. The virtue of this is that it reduces the nonelliptic homogeneous Monge-Ampere equation to a family of elliptic problems, so one can ultimately deduce Theorem 1 from the standard implicit function theorem in Banach spaces. There is also the technical advantage that the method applies to perturbations which are small in $C^{2}$, while the approach of [9] would presumeably need a much larger number of derivatives.

Our second result, which is another consequence of this alternative geometric interpretation of the Problem, is a negative one:

Theorem 2. There are functions $F$ for which Problem 1 does not have a smooth solution.

Thus the strongest analogue of the loop-group factorisation result (Theorem 8.1.1 in [10]) fails, but one may still hope that much more can be said about the nature of the singularities of Chen's solutions (and Chen has already shown, in [2], that the $C^{1,1}$ solutions give useful geometric information). 
In Section 2 we set up the alternative geometric interpretation of the problem using holomorphic discs. This involves a number of steps, but each is essentially elementary. Our treatment here is self-contained and we have intentionally presented the arguments in a rather concrete, co-ordinate based, notation with the aim of avoiding too much abstraction. In Section (2.2) we explain how the arguments can be cast in a more conceptual form using the theory developed in [5], [6]. With this alternative interpretation in place, attenton turns to the corresponding linearised problem, describing the deformations of each disc. In Section 3 we introduce a notion of "superregularity" which implies that the discs appearing in our family are "regular" in the ordinary sense of nonlinear Fredholm theory, and thus that the discfamilies deform in a simple way. The proofs of the main Theorems 1 and 2 are given in Section 4. The proof of Theorem 2 involves the construction of a holomorphic disc and boundary conditions which are not "super-regular".

One can hope to extend the techniques of this paper in two directions. First, one can take the ideas over to the case of the Dirichlet problem over the cylinder which is relevant to the geodesics in the space of Kalher metrics. There is also a variant of this where one considers the punctured disc, with some conditions over the puncture, which arises when one studies geodesic rays in the space of Kahler metrics. Second, one can try to describe more precisely when a smooth solution to Problem 1 exists, and if it does not the nature of the singularities in Chen's solutions. It seems possible that a solution exists precisely when all the holomorphic discs satisfying the relevant boundary conditions are super-regular and, when there are discs which are not superregular, that these will be important in understanding the singularities. However we will leave all of these possible extensions for the future.

A large part of this work was done while the author was at Stanford University, and thanks are due to the Mathematics Department at Stanford for their hospitality and for the stimulating atmosphere there. The author has benefitted from many valuable discussions with X-X. Chen about these topics.

\section{Alternative formulation of the problem.}

We begin by reviewing the construction, described in [6], of a holomorphic fibre bundle $\pi: \mathcal{W}_{V} \rightarrow V$ with the property that the Kahler metrics in the class $\left[\omega_{0}\right]$ correspond to certain submanifolds of $\mathcal{W}_{V}$. Suppose, in general, that $\left(W, \Theta_{W}\right)$ is any complex symplectic manifold; so $\Theta_{W}$ is a nowhere- 
degenerate holomorphic 2-form on $W$. Let $\Theta_{1}, \Theta_{2}$ be the real and imaginary parts of $\Theta_{W}$. We say that a submanifold $L \subset W$ is an $L S$ submanifold if it is Lagrangian with respect to $\Theta_{1}$ while $\Theta_{2}$ restricts to a non-degenerate symplectic form on $L$. We construct the holomorphic fibre bundle $\mathcal{W}_{V}$ with a complex symplectic structure such that the Kahler metrics on $V$ correspond to the sections whose graphs are LS submanifolds of $\mathcal{W}_{V}$.

We start with a local version of the construction. Let $U$ be an open subset of $V$, for example a ball, such that $\omega_{0}$ has a Kahler potential over $U ; \omega_{0}=i \bar{\partial} \partial \rho$ say. We let $\mathcal{W}_{U}$ be the cotangent bundle of $U$ with its standard complex symplectic structure. In local complex co-ordinates $z_{\alpha}$ on $U$ we parametrise the complex 1 -forms by $\sum \xi_{\alpha} d z_{\alpha}$, so $\left(z_{\alpha} ; \xi_{\beta}\right)$ are local co-ordinates on $T^{*} U$. Then the holomorphic form is given by the usual formula

$$
\Theta=\sum d \xi_{\alpha} d z_{\alpha}
$$

We will compare this with the ordinary real symplectic structure on $T^{*} U$, under the standard isomorphism between complex and real cotangent spaces given by taking a complex linear map from $T U$ to $\mathbf{C}$ to its real part. Write

$$
z_{\alpha}=x_{\alpha}+i y_{\alpha}, \xi_{\alpha}=u_{\alpha}+i v_{\alpha}
$$

Then the real part of the complex 1-form $\sum \xi_{\alpha} d z_{\alpha}$ is $\sum u_{\alpha} d x_{\alpha}-v_{\alpha} d y_{\alpha}$. This means that if we take $\left(x_{1} \ldots, x_{n}, y_{1} \ldots, y_{n}\right)$ as real co-ordinates $q_{j}$ on $U$ then the corresponding coordinates $p_{j}$ are $\left(u_{1} \ldots u_{n},-v_{1}, \ldots,-v_{n}\right)$. Now if we write

$$
\Theta=\Theta_{1}+i \Theta_{2}=\sum\left(d u_{\alpha} d x_{\alpha}-d v_{\alpha} d y_{\alpha}\right)+i \sum\left(d u_{\alpha} d y_{\alpha}+d v_{\alpha} d x_{\alpha}\right),
$$

we see that the real part $\Theta_{1}$ of $\Theta$ is the standard real symplectic form $\sum d p_{j} d q_{j}$ on the cotangent bundle. As such, the sections of $T^{*} U$ whose graphs are Lagrangian with respect to $\Theta_{1}$ correspond to the closed 1-forms on $U$ and hence, if $H^{1}(U)=0$, to the derivatives of real-valued functions. In complex notation, if $\psi$ is a real valued function on $U$ we have a corresponding section with

$$
\xi_{\alpha}=2 \frac{\partial \psi}{\partial z_{\alpha}}=\frac{\partial \psi}{\partial x_{\alpha}}-i \frac{\partial \psi}{\partial y_{\alpha}} .
$$

The pull-back of $\Theta$ by this section is the pure imaginary form

$$
2 \sum_{\alpha, \beta} \frac{\partial^{2} \psi}{\partial z_{\alpha} \partial \bar{z}_{\beta}} d \bar{z}_{\beta} d z_{\alpha}=-2 \bar{\partial} \partial \psi,
$$


so the imaginary part of $\Theta$ is nondegenerate on the section if and only if $i \bar{\partial} \partial \psi$ is a nondegenerate Hermitian form. Thus to a Kahler metric $\omega_{0}+i \bar{\partial} \partial \phi=$ $i \bar{\partial} \partial(\rho+\phi)$ over $U$ we associate the LS section defined as above by the derivative of $\psi=\rho+\phi$.

To construct $\mathcal{W}_{V}$ we take a cover $V=\cup U_{\lambda}$ of $V$ by balls and write $\omega_{0}=i \bar{\partial} \partial \rho_{\lambda}$ over $U_{\lambda}$. Then we glue the cotangent bundles $T^{*} U_{\lambda}$ over the intersections $U_{\lambda} \cap U_{\mu}$ by the additive transition functions $\partial\left(\rho_{\lambda}-\rho_{\mu}\right)$. That is we glue a point $\xi$ in a fibre in $T^{*} U_{\lambda}$ to the point $\xi+\partial\left(\rho_{\mu}-\rho_{\lambda}\right)$ in the corresponding fibre in $T^{*} U_{\mu}$. The transition function respects the holomorphic 2 -forms in the different pieces, so we get a holomorphic form $\Theta$ on the total space $\mathcal{W}_{V}$.

Suppose we are given a function $\phi$ on $V$ such that $\omega_{0}+i \bar{\partial} \partial \phi$ is everywhere positive. We define a section of $\mathcal{W}_{V}$ as above, given by the derivative of $\rho_{\lambda}+\phi$ over $U_{\lambda}$, and this is clearly an LS submanifold of $\mathcal{W}_{V}$. Slightly more is true. Recall that, in general, if $L$ is a Lagrangian submanifold of a real symplectic manifold $M$ the normal bundle of $L$ in $M$ is canonically identified with its cotangent bundle and the infinitesimal deformations of $L$, as a Lagrangian submanifold, correspond to the closed 1-forms. A smooth one-parameter family $L_{t}$ of Lagrangian submanifolds is called an exact deformation if at each time the derivative corresponds to an exact 1-form and this generates an equivalence relation on the set of Lagrangian submanifolds. Now in our case it is clear that the $L S$-submanifolds of $\mathcal{W}_{V}$ obtained by our construction are all in the same exact equivalence class - in the family $\omega_{0}+t i \bar{\partial} \partial \phi$ the $t$-derivative corresponds to the exact 1 -form $d \phi$. We will call the LS-submanifolds in this equivalence class the exact LS-submanifolds. (Of course, this refinement is only relevant if $H^{1}(V) \neq 0$.) Now suppose we have a section of $\pi: \mathcal{W}_{V} \rightarrow V$ whose image is a LS-submanifold. Over each ball $U_{\lambda}$ we write this as the derivative of $\rho_{\lambda}+\phi_{\lambda}$ where $\omega_{0}+i \bar{\partial} \partial \phi_{\lambda}>0$. The transition relation shows that $\phi_{\lambda}-\phi_{\mu}$ is constant on $U_{\lambda} \cap U_{\mu}$ and so defines a Cech cohomology class in $H^{1}(V ; \mathbf{R})$. It is easy to see that this class vanishes if the section is an exact LS-submanifold so in this case, after changing the $\phi_{\lambda}$ by the addition of suitable constants, the section corresponds to a global Kahler form $\omega_{0}+i \bar{\partial} \partial \phi$. So in sum we have shown that the Kahler metrics $\omega_{0}+i \bar{\partial} \partial \phi$ are in one-to-one correspondence with the exact LS graphs in $\mathcal{W}_{V}$. (Locally, there are LS submanifolds which correspond to indefinite Kahler metrics on $V$. But a form $\omega_{0}+i \bar{\partial} \partial \phi$ over the compact manifold $V$ is necessarily positive at the maximum of $\phi$, so there are no indefinite solutions globally.)

The construction of $\mathcal{W}_{V}$ is in a sense a standard matter. Recall that the 
Kahler form defines a Dolbeault cohomology class in $H^{1}\left(T^{*} V\right)$. This defines an extension of vector bundles

$$
0 \rightarrow T^{*} V \rightarrow J \rightarrow \mathcal{O} \rightarrow 0
$$

The complex manifold $\mathcal{W}_{V}$ can be identified with the open subset of the projectivisation

$$
\mathcal{W}_{V}=\mathbf{P}(J) \backslash \mathbf{P}\left(T^{*} V\right),
$$

and the 2-form $\Theta$ is a meromorphic form on $\mathbf{P}(J)$ with a pole of order $n+1$ along $\mathbf{P}\left(T^{*} V\right)$. There is further interpretation of this in the case when the Kahler class $\left[\omega_{0}\right]$ is integral, so there is a holomorphic line bundle $\mathcal{L} \rightarrow V$ with a hermitian connection whose curvature is $-2 \pi i \omega_{0}$. The holomorphic bundle $J$ can then be identified with $J_{1}(L) \otimes L^{-1}$, where $J_{1}(L)$ is the jet bundle of 1-jets of sections of $L$. In this interpretation a Kahler metric $\omega_{0}+$ $i \bar{\partial} \partial \phi$ defines another unitary connection on the fixed holomorphic bundle $\mathcal{L}$ and the section $L_{\phi}$ is given by the projectivisation of the set of horizontal jets, with respect to this connection.

\subsection{Re-formulation.}

Let $F$ be a smooth function on $\partial D \times V$, as in the statement of Problem 1 . For each $\tau \in \partial D$ the function $F_{\tau}(x)=F(\tau, x)$ defines a Kahler metric and hence an LS submanifold $\Lambda_{\tau}$ of $\mathcal{W}_{V}$. The main result of this section is

Theorem 3. There is a solution $\Phi$ of Problem 1 if and only if there is a smooth family of holomorphic discs $g_{x}: D \rightarrow \mathcal{W}_{V}$ parametrised by $x \in V$ with

1. $\pi\left(g_{x}(0)\right)=x \in V$;

2. for each $\tau \in \partial D$ and each $x \in V$,

$$
g_{x}(\tau) \in \Lambda_{\tau}
$$

3. for each $\tau \in D$ the map $x \mapsto \pi\left(g_{x}(\tau)\right)$ is a diffeomorphism from $V$ to $V$.

To be precise we mean that there is a map $G: D \times V \rightarrow \mathcal{W}_{V}$ which is smooth as a map on the manifold-with-boundary $V \times D$, which is holomorphic over the interior in the $D$-variable and which satisfies

$$
G(0, x)=x, G(\tau, x) \in \Lambda_{\tau} .
$$


Here of course the two notations are related by setting

$$
g_{x}(\tau)=G(\tau, x) .
$$

The essence of our approach is to think of the map $G$ in two ways; either as a family of maps $g_{x}$, as above, or as a family of maps

$$
\gamma_{\tau}: V \rightarrow \mathcal{W}_{V}
$$

with $\gamma_{\tau}(x)=G(\tau, x)$.

We will now proceed to give a direct proof of Theorem 3. To begin, suppose we have a solution $\Phi$ of Problem 1. Changing the choice of $\omega_{0}$, there is no loss in supposing that $\Phi(0, x)=0$ for all $x$. The Monge-Ampère condition $\left(\Omega_{0}+i \bar{\partial} \partial \Phi\right)^{n+1}=0$ implies that the Hermitian form $\Omega_{0}+i \bar{\partial} \partial \Phi$ has a non-trivial kernel at each point and the hypothesis that the form is strictly positive in the vertical slices implies that the kernel has complex dimension 1 . Since the 2 -form is closed, these kernels define a foliation transverse to the vertical slices, whose leaves are complex curves in $D \times V$. The disc $D$ is simply connected so each of these leaves is a copy of $D$ - the graph of a holomorphic map from $D$ to $V$. Thus for each $x$ in $V$ we have a holomorphic map $f_{x}: D \rightarrow V$, with $f_{x}(0)=x$, whose graph is the leaf in $D \times V$ passing through $(0, x)$. If we write $f_{x}(\tau)=\sigma_{\tau}(x)$ we get a family of "monodromy" diffeomorphisms $\sigma_{\tau}: V \rightarrow V$, with $\sigma_{0}=\operatorname{Id}_{V}$.

Lemma 1. For each $\tau \in D, \sigma_{\tau}^{*}\left(\omega_{0}+i \bar{\partial} \partial \phi_{\tau}\right)=\omega_{0}$.

To prove this we show that the $\tau$-derivative of $\sigma_{\tau}^{*}\left(\omega_{0}+i \bar{\partial} \partial \phi_{\tau}\right)$ vanishes. So let $v$ be a tangent vector on the disc at a point $\tau$ and let $\tilde{v}$ be the unique tangent vector in the kernel of $\Omega_{0}+i \bar{\partial} \partial \Phi$ at $(\tau, x) \in D \times V$ which projects to $v$. Then the derivative of $\sigma_{\tau}^{*}\left(\omega_{0}+i \bar{\partial} \partial \phi_{\tau}\right)$ along $v$, is the pull-back by $\sigma_{\tau}^{*}$ of the restriction of the Lie derivative along $\tilde{v}$ of $\Omega_{0}+i \bar{\partial} \partial \Phi$ to the slice $\{\tau\} \times V$. But this vanishes by the usual "homotopy" formula for the Lie derivative on forms, since $\Omega_{0}+i \bar{\partial} \partial \Phi$ is closed and $\tilde{v}$ lies in its kernel.

Now for each fixed $\tau$ in $D$ we have a Kahler form $\omega_{0}+i \bar{\partial} \partial \phi_{\tau}$ on $V$ and hence a section $s_{\tau}: V \rightarrow \mathcal{W}_{V}$ whose image is an exact LS graph $\Lambda_{\tau}$ in $\mathcal{W}_{V}$. We define $G: D \times V \rightarrow \mathcal{W}_{V}$ by

$$
G(\tau, x)=s_{\tau}\left(\sigma_{\tau}(x)\right) .
$$

Using the notation above, thinking of either of the variables as parameters, we will write

$$
G(x, \tau)=g_{x}(\tau)=\gamma_{\tau}(x) .
$$

Thus to sum up we have 
1. For each fixed $x, g_{x}: D \rightarrow \mathcal{W}_{V}$ is a lift of the holomorphic map $f_{x}: D \rightarrow V$ over the projection $\pi: \mathcal{W}_{V} \rightarrow V$;

2. For each fixed $\tau, \gamma_{\tau}: V \rightarrow \mathcal{W}_{V}$ is a diffeomorphism from $V$ onto an LS graph $\Lambda_{\tau} \subset \mathcal{W}_{V}$ and $\gamma_{\tau}^{*}(\Theta)=-2 \omega_{0}$.

Here the formula for $\gamma_{\tau}^{*}(\Theta)$ follows from (1) and Lemma 1.

The following Lemma completes the proof of one half of Theorem 3.

Lemma 2. The map $G$ above is holomorphic in the D-variable, that is, for each fixed $x \in V, g_{x}: D \rightarrow \mathcal{W}_{V}$ is holomorphic

The result is local so we may as well suppose that $g_{x}$ maps into a subset of the form $T^{*} U \subset \mathcal{W}_{V}$, and choose local co-ordinates $z_{\alpha}$ on $U$. We know that the projection to the base gives the holomorphic map $f_{x}$ so it suffices to consider the fibre component of $T^{*} U$. By definition this is

$$
\sum \frac{\partial \Psi}{\partial z_{\alpha}} d z_{\beta}
$$

where $\Psi=\Phi+\rho$. So we need to show that

$$
\frac{\partial}{\partial \bar{\tau}} \frac{\partial}{\partial z_{\alpha}} \Psi\left(\tau, f_{x}(\tau)\right)
$$

vanishes. By the chain rule this is

$$
\frac{\partial^{2} \Psi}{\partial \bar{\tau} \partial z_{\alpha}}+\sum_{\beta} \frac{\partial^{2} \Psi}{\partial z_{\alpha} \partial z_{\beta}} \frac{\partial f_{x}^{(\beta)}}{\partial \tau}
$$

where $f_{x}^{(\beta)}$ are the components of the map $f_{x}$ in these local co-ordinates. But this is the same as $\Omega_{0}+i \bar{\partial} \partial \Phi$ evaluated on the two tangent vectors in $D \times V$ :

$$
\frac{\partial}{\partial \tau}+\sum_{\beta} \frac{\partial f_{x}^{(\beta)}}{\partial \tau} \frac{\partial}{\partial z_{\beta}}, \frac{\partial}{\partial z_{\alpha}},
$$

and this does indeed vanish since, by the definition of $f_{x}$, the first tangent vector lies in the kernel of $\Omega_{0}+i \bar{\partial} \partial \Phi$.

To sum up so far, we have shown that starting from a solution $\Phi$ of Problem 1 one can construct a family of holomorphic discs $g_{x}: D \rightarrow \mathcal{W}_{V}$ satisfying the conditions of Theorem 3 . We now move on to the reverse direction. 
Proposition 1. Suppose given a function $F$ on $\partial D \times V$ as in Problem 1. Suppose we have a map $G: D \times V \rightarrow \mathcal{W}_{V}$ and write $G(\tau, x)=g_{x}(\tau)=\gamma_{\tau}(x)$ as before. Suppose that

1. $G(0, x)=x$ for all $x \in V$;

2. For each fixed $x, g_{x}$ is a holomorphic map from $D$ to $\mathcal{W}_{V}$;

3. For each fixed $\tau, \gamma_{\tau}$ maps $V$ diffeomorphically to an exact $L S$ graph in $\mathcal{W}_{V}$

4. For $\tau \in \partial D$ the image of $\gamma_{\tau}$ is the exact $L S$ graph $L_{\tau}$ defined by the boundary data.

Then $G$ arises from a (unique) solution $\Phi$ of Problem 1 via the construction above.

To prove this Proposition, recall that $\Theta$ is a closed holomorphic 2-form on $\mathcal{W}_{V}$ so that its real and imaginary parts $\Theta_{1}, \Theta_{2}$ are closed real 2-forms and for any tangent vectors $\xi, \eta$ at a point in $\mathcal{W}_{V}$ :

$$
\Theta_{2}(\xi, \eta)=\Theta_{1}(I \xi, \eta)
$$

where $I$ denotes the usual endomorphism of the tangent space defined by the complex structure. We consider the pull-backs $G^{*}\left(\Theta_{1}\right), G^{*}\left(\Theta_{2}\right)$ to closed 2 -forms on $D \times V$. For clarity let us take (real) local co-ordinates $q_{j}$ in $V$ and write $\tau=u+i v$ where $u, v$ are real. Then the fact that each $\gamma_{\tau}$ maps to a $\Theta_{1^{-}}$ Lagrangian submanifold of $V$ means that $G^{*}\left(\Theta_{1}\right)$ does not contain any terms in $d q_{i} d q_{j}$. The fact that $g_{x}$ is holomorphic, combined with (2), means that neither $G^{*}\left(\Theta_{1}\right)$ nor $G^{*}\left(\Theta_{2}\right)$ contains a term in $d u d v$. Further, (2) implies that the $d u d q_{j}$ component of $G^{*}\left(\Theta_{2}\right)$ is equal to the $d v d q_{j}$ component of $G^{*}\left(\Theta_{1}\right)$ and the $d v d q_{j}$ component of $G^{*}\left(\Theta_{2}\right)$ is minus the $d u d q_{j}$ component of $G^{*}\left(\Theta_{1}\right)$. So we can write

$$
\begin{aligned}
G^{*}\left(\Theta_{1}\right) & =\sum a_{j} d u d q_{j}+b_{j} d v d q_{j}, \\
G^{*}\left(\Theta_{2}\right) & =\sum b_{j} d u d q_{j}-a_{j} d v d q_{j}+\sum r_{i j} d q_{i} d q_{j} .
\end{aligned}
$$

Now the fact that $G^{*}\left(\Theta_{1}\right)$ is closed implies that

$$
\begin{aligned}
& \frac{\partial a_{j}}{\partial q_{i}}=\frac{\partial a_{i}}{\partial q_{j}} \\
& \frac{\partial b_{j}}{\partial q_{i}}=\frac{\partial b_{i}}{\partial q_{j}}
\end{aligned}
$$


while the fact that $G^{*}\left(\Theta_{2}\right)$ is closed implies that

$$
\begin{aligned}
\frac{\partial r_{i j}}{\partial u} & =\frac{\partial b_{j}}{\partial q_{i}}-\frac{\partial b_{i}}{\partial q_{j}} \\
\frac{\partial r_{i j}}{\partial v} & =\frac{\partial a_{i}}{\partial q_{j}}-\frac{\partial a_{j}}{\partial q_{i}} .
\end{aligned}
$$

So it follows that $r_{i j}$ is independent of $u, v$. Thus $G^{*}\left(\Theta_{2}\right)=-2 \Omega_{0}$ is constant along the horizontal slices in $D \times V$.

Now the hypothesis that the $\gamma_{\tau}$ map diffeomorphically to graphs in $\mathcal{W}_{V}$ implies that the composites $\pi \circ \gamma_{\tau}: V \rightarrow V$ are diffeomorphisms. Let $\sigma_{\tau}: V \rightarrow V$ be the inverse diffeomorphisms. So by assumption $\sigma_{0}$ is the identity map. Let $f_{x}$ be the holomorphic map $\pi \circ g_{x}: D \rightarrow V$. Then the graphs of the family of holomorphic maps $f_{x}$ make up a foliation $\mathcal{F}$ of $D \times V$ by holomorphic curves, transverse to the vertical slices. Define $R: D \times V \rightarrow D \times V$ by $R(\tau, x)=\left(\tau, \sigma_{\tau}(x)\right)$. Thus $R$ maps the trivial product foliation of $D \times V$ to the foliation $\mathcal{F}$, or in other words the diffeomorphisms $\sigma_{\tau}$ give the monodromy of the foliation $\mathcal{F}$.

For each fixed $\tau$ the image of $\gamma_{\tau}$ is an LS graph in $\mathcal{W}_{V}$ and so corresponds to a Kahler metric $\omega_{\tau}=\omega_{0}+i \bar{\partial} \partial \tilde{\phi}_{\tau}$ say, where $\tilde{\phi}_{\tau}$ is unique up to the addition of a constant. We know from the above that $\gamma_{\tau}^{*}\left(\Theta_{2}\right)=-2 \omega_{\tau}$, so $\sigma_{\tau}^{*}\left(\omega_{\tau}\right)=\omega_{0}$.

Now at each point $(\tau, x)$ of $D \times V$ there is a unique Hermitian form $\Omega$ on the tangent space to $D \times V$ with the two properties

1. The restriction of $\Omega$ to the vertical slice through $(\tau, x)$ is equal to $\omega_{\tau}$.

2. The tangent space to the leaf of the foliation $\mathcal{F}$ at this point is the kernel of $\Omega$.

Thus we have a $(1,1)$-form $\Omega$ on $D \times V$. We claim next that $\Omega$ is closed. The restriction of $d \Omega$ to the vertical slices vanish by the first property above so it suffices to show that the contraction $i_{X}(d \Omega)$ is zero, where $X$ is a vector field tangent to the foliation. But the fact, established in the preceding paragraph, that $\sigma_{\tau}^{*}\left(\omega_{\tau}\right)=\omega_{0}$ implies that Lie derivative of $\Omega$ along $X$ is zero. This Lie derivative is $d\left(i_{X}(\Omega)\right)+i_{X}(d \Omega)$ and $i_{X}(\Omega)$ vanishes by the second property. So we conclude that $\Omega$ is a closed form of type $(1,1)$ on $V \times D$.

The final step in the proof is to write $\Omega=\Omega_{0}+i \bar{\partial} \partial \Phi$ for a suitable function $\Phi$. This is a version of the $\bar{\partial} \partial$-lemma, which brings in the condition of exactness. Let us suppose first that $H^{1}(V ; \mathbf{R})=0$. We can certainly 
choose a function $\tilde{\Phi}$ such that $\chi=\Omega-\left(\Omega_{0}+i \bar{\partial} \partial \tilde{\Phi}\right)$ vanishes on all vertical slices, and by the given boundary conditions we may suppose that $\tilde{\Phi}=F$ on the boundary of $D \times V$. In local co-ordinates we can write $\chi$ as

$$
\sum \mu_{\alpha} d z_{\alpha} d \tau+\bar{\mu}_{\alpha} d \bar{z}_{\alpha} d \bar{\tau}+f d \tau d \bar{\tau}
$$

The fact that $\chi$ is closed means that

$$
\frac{\partial \mu_{\alpha}}{\partial \bar{z}_{\beta}}=0
$$

Globally, $\sum \mu_{\alpha} d z_{\alpha}$ defines a holomorphic 1-form $S$ on $V$. But if, as we are assuming, the cohomology group $H^{1}(V)$ vanishes there are no non-trivial holomorphic 1-forms so $S=0$. Then, using again the fact that $\chi$ is closed, we have

$$
\frac{\partial f}{\partial z_{\alpha}}=0
$$

so $f$ is a function of $\tau$. Then we solve a linear boundary value problem on the disc to find a function $h(\tau)$, vanishing on the boundary circle and with $i \bar{\partial} \partial h=f d \tau d \bar{\tau}$. The function $\Phi=\tilde{\Phi}-h$ is the solution we seek.

To deal with the subtlety involving exactness in the case when $H^{1}(V)$ is non-trival we go back to $(5),(6)$. We saw that the 1 -forms

$$
\lambda_{1}=\sum a_{j} d q_{j}, \lambda_{2}=\sum b_{j} d q_{j}
$$

are closed on $V$. The discussion there was local but $\lambda_{1}, \lambda_{2}$ are globally defined, as families of closed 1-forms on $V$ parametrised by $\tau$. The condition that the family of LS submanifolds are all exact means precisely that we can find functions $s_{1}, s_{2}$ with $d s_{i}=\lambda_{i}$. Following through the construction, one finds that the holomorphic 1-form $S=\sum \mu_{\alpha} d z_{\alpha}$ is just the pull-back under $\sigma_{\tau}$ of $d\left(s_{1}+i s_{2}\right)$. So $S$ is an exact holomorphic 1-form and hence vanishes. Then the proof can proceed as before.

The next Lemma is the crucial step in completing the proof of Theorem 3.

Lemma 3. Suppose given boundary data $F$ as in Problem 1 and that $G$ : $D \times V \rightarrow \mathcal{W}_{V}$ is a map such that for each $x, g_{x}: D \rightarrow \mathcal{W}_{V}$ is holomorphic and for $\tau \in \partial D, \gamma_{\tau}$ maps $V$ diffeomorphically to the $L S$ graph $L_{\tau}$ defined by the boundary data. Then for each $\tau$ in $D$ the map $\gamma_{\tau}$ is an immersion of $V$ whose image is an exact $\Theta_{1}$-Lagrangian immersed submanifold on which $\Theta_{2}$ is non-degenerate. 
To prove this we again consider the pull-back $G^{*}(\Theta)$, a 2-form with complex-co-efficients on $D \times V$. We can decompose 2 -forms on the product into three pieces: let $G^{*}(\Theta)_{V}$ denote the component of $G^{*}(\Theta)$ in $\Lambda^{2} T^{*} V \subset$ $\Lambda^{2} T^{*}(D \times V)$. Thus we can think of $G^{*}(\Theta)_{V}$ as a family of complex 2-forms on $V$ parametrised by $\tau \in D$. The key point is that, since $\Theta$ is a holomorphic form on $\mathcal{W}_{V}$ and the maps $g_{x}$ are holomorphic, this family is holomorphic in $\tau$. To see this we write, in the same notation as before,

$$
\begin{aligned}
G^{*}\left(\Theta_{1}\right) & =\sum a_{j} d u d q_{j}+b_{j} d v d q_{j}+\sum s_{i j} d q_{i} d q_{j} \\
G^{*}\left(\Theta_{2}\right) & =\sum b_{j} d u d q_{j}-a_{j} d v d q_{j}+\sum r_{i j} d q_{i} d q_{j} .
\end{aligned}
$$

The fact that $G^{*}\left(\Theta_{1}\right)$ is closed implies that, in place of $(5),(6)$ we have,

$$
\begin{aligned}
\frac{\partial s_{i j}}{\partial u} & =\frac{\partial a_{i}}{\partial q_{j}}-\frac{\partial a_{j}}{\partial q_{i}} \\
\frac{\partial s_{i j}}{\partial v} & =\frac{\partial b_{i}}{\partial q_{j}}-\frac{\partial b_{j}}{\partial q_{i}},
\end{aligned}
$$

with the same set of equations (7),(8) as before. This means that

$$
\left(\frac{\partial}{\partial u}+\sqrt{-1} \frac{\partial}{\partial v}\right)\left(r_{i j}+\sqrt{-1} s_{i j}\right)=0,
$$

which is just the statement that $G^{*}(\Theta)_{V}$ is holomorphic in $\tau$. We now use the elementary fact that a holomorphic function on the disc which is real on the boundary must be a constant. By hypothesis the form $i G^{*}(\Theta)_{V}$ is real when $\tau$ lies in the boundary of the disc. Applying this fact to the co-efficients of the form we we see that the real part of $G^{*}(\Theta)_{V}$ vanishes everywhere-so in fact the $s_{i j}$ are all zero- and the imaginary part is constant in $\tau$. In other words $\gamma_{\tau}^{*}\left(\Theta_{1}\right)=0$ and $\gamma_{\tau}^{*}\left(\Theta_{2}\right)=\theta$ say. But, again by hypothesis, when $\tau$ is in the boundary $\gamma_{\tau}$ maps diffeomorphically to the LS submanifold $\Lambda_{\tau}$ on which $\Theta_{2}$ is non-degenerate. So $\theta$ is a nondegenerate 2-form on $V$. This means that, for each $\tau, \gamma_{\tau}$ must be an immersion. The only remaining thing to check is the exactness condition. We consider the family of complex 1 -forms $\sigma_{\tau}$ on $V$, parametrised by $\tau$,

$$
\sigma_{\tau}=\sum\left(a_{j}+i b_{j}\right) d q_{j}
$$

The hypotheses imply that for fixed $\tau, \sigma_{\tau}$ is closed on $V$, and the family is holomorphic in $\tau$. So we get a holomorphic map $\left[\sigma_{\tau}\right]$ from the disc into $H^{1}(V: \mathbf{C})$. Let $c(t)$ be any path in the disc. By definition, the 1-parameter 
family $g_{c(t)}$ map into the same exact equivalence class of LS submanifolds if the imaginary part of $c^{\prime}(t) \sigma_{c(t)}$ is zero. Thus the hypothesis that the $g_{\tau}$ map into exact LS submanifolds when $\tau$ lies in the boundary of the disc means that $\tau\left[\sigma_{\tau}\right]$ is pure imaginary when $\tau$ lies in this boundary. Hence $\tau\left[\sigma_{\tau}\right]$ is constant, as above, and taking $\tau=0$ we see that the constant must be zero. So we conclude that $\sigma_{\tau}$ is exact for each $\tau \in D$, and this means that the images of the maps $g_{\tau}$ are exact.

Combining Proposition 1 with Lemma 3, we have now completed the proof of Theorem 3 .

\subsection{Complexifications of symplectomorphism groups.}

So far we have presented the arguments in a somewhat explicit way, emphasising calculations in local co-ordinates. We will now explain how Theorem 3 can be obtained by a more conceptual approach, avoiding explicit calculations. For simplicity we suppose that $H^{1}(V)=0$ and that $V$ has no non-trivial holomorphic automorphisms. (The first of these assumptions means that we do not need to bother about the exactness condition.) Let $(P, \omega)$ be a symplectic manifold. Recall, following the discussion in Section 4 of [5], that by a complexification of the group SDiff ${ }_{\mathrm{P}, \omega}$ of symplectomorphisms of $(P, \omega)$ we mean an infinite-dimensional complex manifold $\mathcal{Z}$ with a free action of SDiff ${ }_{\mathrm{P}, \omega}$ such that the tangent space of $\mathcal{Z}$ at a point $z$ is the direct sum $T O_{z} \oplus I T O_{z}$ where $O_{z}$ is the orbit of SDiff ${ }_{\mathrm{P}, \omega}$ through $z$ and $I$ is the action of complex multiplication on tangent vectors of $\mathcal{Z}$. In the case when $(P, \omega)=\left(V, \omega_{0}\right)$ is Kahler it is explained in [5] that there is such a complexification $\mathcal{X}$ given by the set of pairs $(f, \phi)$ where $\omega_{\phi}=\omega_{0}+i \bar{\partial} \partial \phi>0$ and $f: V \rightarrow V$ is such that $f^{*}\left(\omega_{\phi}\right)=\omega_{0}$. (Here, $\phi$ should really be considered as an element of $C^{\infty}(V)$ modulo the constants.) The complex structure on $\mathcal{X}$ could be seen in two ways:

1. Via the map $(f, \phi) \mapsto f^{*}(I)$ which embeds $\mathcal{X}$ in the space of almostcomplex structures on $V$, compatible with $\omega_{0}$.

2. Via the map $(f, \phi) \mapsto f$ which embeds $\mathcal{X}$ in the space $\operatorname{Maps}(V, V)$, which has a complex structure defined by that on $V$ (regarded as the target space).

Suppose now that $\left(W, \Theta_{W}\right)$ is a complex symplectic manifold and $(P, \omega)$ is a real symplectic manifold with $\operatorname{dim}_{\mathbf{R}} P=\operatorname{dim}_{\mathbf{C}} W$. We consider the set $\mathcal{L}$ of maps (in a given isotopy class) $\lambda: P \rightarrow W$ such that

$$
\lambda^{*}\left(\Theta_{W}\right)=-2 \omega .
$$


This set of "parametrised LS submanifolds" was discussed in [6], where it was observed that $\mathcal{L}$ is a complexification of $\operatorname{SDiff}_{\mathrm{P}, \omega}$. The point is that the equation (9) is a holomorphic equation for $\lambda$, where we take the obvious complex structure on $\operatorname{Maps}(P, \mathcal{W})$ defined by that on $\mathcal{W}$. Now the crucial thing is that, when $(P, \omega)=\left(V, \omega_{0}\right)$ and $W=\mathcal{W}_{V}$, these two constructions are compatible. More precisely, we restrict attention to the open subset of maps $\lambda$ from $V$ to $\mathcal{W}_{V}$ whose images are LS graphs (which does not affect the formalism). Then we have seen that such a graph defines a Kahler metric $\omega_{\phi}$ on $V$ and for $\lambda \in \mathcal{L}$ the composite $f=\pi \circ \lambda: V \rightarrow V$ satisfies $f^{*}\left(\omega_{\phi}\right)=\omega_{0}$. This induces an SDiff-equivariant bijection $\sigma$ from $\mathcal{L}$ to $\mathcal{X}$, and $\sigma$ is obviously holomorphic since the projection $\pi: \mathcal{W}_{V} \rightarrow V$ is (using the second description above) of the complex structure on $\mathcal{X}$.

It is easy to deduce Theorem 3 from this alternative description of the complexification. For, as explained in [5], a solution of Problem 1 corresponds to a holomorphic map $\tilde{\Gamma}$ from the disc into $\mathcal{X}$ such that $\tilde{\Gamma}(\tau)$ lies in the SDiff-orbit defined by the given boundary data, for $\tau \in \partial D$. By the discussion above, this is the same as a holomorphic map $\Gamma: D \rightarrow \mathcal{L}$ such that $\Gamma(\tau)$ maps to the LS graph $\Lambda_{\tau}$ defined by the boundary data, for $\tau \in \partial D$. But a holomorphic map from $D$ to $\operatorname{Maps}\left(V, \mathcal{W}_{V}\right)$ is precisely a family of holomorphic discs in $\mathcal{W}_{V}$ parametrised by $V$ so we immediately arive at the formulation of Proposition 1. The crucial Lemma 3, which says that it suffices to find a map into the ambient $\operatorname{space} \operatorname{Maps}\left(V, \mathcal{W}_{V}\right)$, satisfying the given boundary conditions, is a manifestation of the fact that $\mathcal{L}$ is a complex submanifold of $\operatorname{Maps}\left(V, \mathcal{W}_{V}\right)$. This means that any holomorphic map of the disc into $\operatorname{Maps}\left(V, \mathcal{W}_{V}\right)$ which maps the boundary into $\mathcal{L}$ must map the whole disc into $\mathcal{L}$.

\section{Families of holomorphic discs.}

We will now review the relevant theory of holomorphic maps with boundary conditions. Suppose $Z$ is a complex manifold of complex dimension $m$ and $X \subset Z$ is a totally real submanifold. Recall that this means that $X$ has real dimension $m$ and at each point of $X$

$$
T X \cap I(T X)=\{0\} \subset T Z \text {. }
$$

We consider holomorphic maps from the disc $D$ to $Z$ which map $\partial D$ into $X$. The crucial thing is that this is an elliptic boundary value problem. There is an extensive literature about this problem, from the point of view

of complex analysis [1] and symplectic geometry [7]. (In the symplectic 
geometry literature it is often assumed that the submanifold $X$ is Lagrangian with respect to a symplectic form which "tames" the complex structure but this condition is only relevant to global compactness questions, and not to the local deformation theory which is our concern here.) There is a linearised problem associated to any such holomorphic map $\psi:(D, \partial D) \rightarrow(Z, X)$. We pull back the tangent bundle of $Z$ to get a holomorphic bundle $E_{\psi}$ over the disc with a Cauchy-Riemann operator

$$
\bar{\partial}_{\psi}: \Omega_{D}^{0}(E) \rightarrow \Omega_{D}^{0,1}(E) .
$$

The tangent space of $X$ defines a real sub-bundle $E_{\mathbf{R}}$ of $E$ over the boundary of the disc and so a projection $p:\left.E\right|_{\partial D} \rightarrow E / E_{\mathbf{R}}$. The linearised equation associated to the mapping problem is the linear elliptic boundary value problem

$$
\bar{\partial}_{\psi} s=0 ; p\left(\left.s\right|_{\partial D}\right)=0 \text {. }
$$

The solution space $H_{\psi}$ of this linear equation is a finite dimensional real vector space. In the familiar way there is a Fredholm index ind $(\psi)$ associated to this problem

$$
\text { ind }(\psi)=\operatorname{dim} \mathrm{H}-\operatorname{dim} \mathrm{H}^{\prime},
$$

where $H^{\prime}$ is the finite-dimensional space of solutions of the adjoint boundary value problem. Thus elements of $H^{\prime}$ are holomorphic, $E_{\psi^{-}}^{*}$ valued 1-forms $\lambda(\tau) d \tau$ over the disc such that at each point $\tau$ of the boundary

$$
i \tau \lambda(\tau) \in \operatorname{Ann}\left(E_{\mathbf{R}}\right)
$$

The index is a homotopy invariant of the data consisting of the bundle $E_{\psi}$ over the disc and the totally-real sub-bundle $E_{\mathbf{R}}$ over the boundary. We say that the map $\psi$ is a regular solution of the nonlinear boundary value problem if $H^{\prime}=0$.

A complication in these mapping problems arises from the fact that a single (non-constant) map $\psi$ leads to 3 -dimensional family by merely composing with the automorphisms of the disc. Thus we have a canonical injection from $\mathfrak{s l}_{2}(\mathbf{R})$ into $H_{\psi}$. To get around this we consider a situation in which there is a fixed holomorphic map $k: Z \rightarrow \mathbf{C}$ which maps $X$ to the boundary circle $\partial D$. Then we consider "normalised" maps $\psi$ such that $k \circ \psi$ is the identity map on the disc. The derivative of $k$ defines a splitting of the injection and hence a canonical direct sum decomposition

$$
H_{\psi}=\mathfrak{s l}_{2}(\mathbf{R}) \oplus H_{\psi, 0},
$$


say.

Now suppose $M$ is a compact, connected manifold parametrising a family of normalised solutions to the boundary value problem. That is, we have a smooth map $B: D \times M \rightarrow Z$ such that for each $m \in M$ the map $\psi_{m}(\tau)=B(\tau, m)$ is holomorphic in $\tau$, maps $\partial D$ to $X$ and $k \circ \psi_{m}=\operatorname{Id}_{D}$. For each $m \in M$ we get a deformation map

$$
R_{m}: T M_{m} \rightarrow H_{\psi_{m}, 0},
$$

reflecting the fact that $H_{\psi_{m}}$ is the space of solutions of the linearised problem.

\section{Proposition 2.}

1. Suppose that for each $m \in M$ the map $\psi_{m}$ is regular. Let $X^{\prime}$ be another totally real submanifold of $Z$ with $k\left(X^{\prime}\right) \subset \partial D$. If $X^{\prime}$ is sufficiently close to $X$ in the $C^{1}$ topology, there is a map $B^{\prime}: D \times M \rightarrow Z, C^{1}$ close to $B$, parametrising a family of normalised maps $\psi_{m}^{\prime}:(D, \partial D) \rightarrow$ $\left(Z, X^{\prime}\right)$.

2. Suppose in addition that $R_{m}$ is an isomorphism for all $m \in M$. Then, if $X^{\prime}$ is sufficiently close to $X$ in the $C^{1}$ topology, the map $B^{\prime}$ is unique up to a diffeomorphism of $M$ and any normalised holomorphic map $\psi^{\prime}$ with $\psi^{\prime}(\partial D) \subset X^{\prime}$ which is close in $C^{1}$ to some map $\psi_{m}$ is equal to $\psi_{m^{\prime}}^{\prime}$ for some $m^{\prime} \in M$.

\section{Remarks.}

1. In essence this Proposition asserts that compact families of regular, normalised, solutions of the boundary value problem are stable under small perturbations. We refer to the literature cited above for the proof, which is essentially routine once one has set up the mapping problem as a nonlinear Fredholm equation.

2. When we say that $X^{\prime}$ is close to $X$ in the $C^{1}$ topology, we mean that $X^{\prime}$ is the image of a smooth map from $X$ to $Z$ which is close in $C^{1}$ to the inclusion map.

3. When we say, for example, that $B^{\prime}$ is $C^{1}$-close to $B$, we mean that the $C^{1}$ distance between $B$ and $B^{\prime}$ is bounded by a function $\delta$ of the $C^{1}$ distance from $X$ and $X^{\prime}$, with $\delta(s) \rightarrow 0$ as $s \rightarrow 0$. (In fact one can take $\delta(s)=C s$. ) 
To apply this theory to our problem, we let $Z$ be the product $\mathcal{W}_{V} \times \mathbf{C}$ and $k: Z \rightarrow \mathbf{C}$ be the projection map. Thus normalised holomorphic maps from $D$ to $Z$ are just the graphs of holomorphic maps from $D$ to $\mathcal{W}_{V}$. Given boundary data $F$ as in Problem 1, we define

$$
X=X_{F}=\left\{(w, \tau) \in \mathcal{W}_{V} \times \partial D: w \in \Lambda_{\tau}\right\}
$$

the LS graph defined by the boundary data. Then the normalised holomorphic maps from $(D, \partial D)$ to $(Z, X)$ precisely correspond to the graphs of a map $g: D \rightarrow \mathcal{W}_{V}$ of the kind considered in Theorem 3. (It is a matter of choice whether one sets up the problem in the way we have done, taking the product with an extra variable, or stays with maps into $\mathcal{W}_{V}$ but with " $\tau$-dependent" boundary conditions.)

Lemma 4. The submanifold $X_{F}$ is totally real in $\mathcal{W}_{V} \times \mathbf{C}$.

For if $\xi$ and $I \xi$ are both tangent vectors to $X_{F}$ at a point $(w, \tau)$ one sees first, by considering the projection to $\mathbf{C}$, that they lie in the tangent space to $\Lambda_{\tau}$. Now the restriction of $\Theta_{2}$ to $\Lambda_{\tau}$ is nondegenerate so there is some $\eta$ tangent to $\Lambda_{\tau}$ such that $\Theta_{2}(\xi, \eta) \neq 0$. But then

$$
\Theta_{1}(I \xi, \eta)=-\Theta_{2}(\xi, \eta) \neq 0
$$

which contradicts the fact that $\Lambda_{\tau}$ is $\Theta_{1}$-Lagrangian.

Remark. One should be careful to distinguish here between the Lagrangian nature of $\Lambda_{\tau}$ with respect to $\Theta_{1}$ - the real part of a holomorphic symplectic form - and the Lagrangian submanifolds, often considered in such discmappping problems, which are Lagrangian with respect to a Kahler form.

We are thus in exactly the position considered above. If we have solution $\Phi$ to Problem 1 with some boundary data $F$ we get a family of normalised holomorphic maps $\psi_{x}$ from $(D, \partial D)$ to $\left(Z, X_{F}\right)$, parametrised by the compact manifold $V$ :

$$
\psi_{x}(\tau)=\left(\tau, g_{x}(\tau)\right)
$$

The crucial result we need, which is established in the next subsection, is:

Proposition 3. Each of the maps $\psi_{x}$ arising from a smooth solution $\Phi$ of Problem 1 is regular with index $2 n$. 


\subsection{Analysis of the linearised problem.}

We introduce a class of a linear boundary value problems: at the end of this subsection we will see how the linearised mapping problem above can be put in this form. Suppose $A$ is a smooth map from the circle $\partial D$ to the strictly positive definite Hermitian $n \times n$ matrices, and $S$ is a smooth map from the circle to the symmetric complex $n \times n$ matrices. We consider pairs of $\mathbf{C}^{n}$-valued functions $(u, v)$ over the disc satisfying the linear boundary condition

$$
v(\tau)=S_{\tau}(u(\tau))+A_{\tau}(\bar{u}(\tau))
$$

for $\tau \in \partial D$. Here we have written the arguments of $S$ and $A$ as a subscript for clarity. We have an operator

$$
\bar{\partial}_{S, A}: L_{S, A}^{2,1}(D) \rightarrow L^{2}(D)
$$

where $L_{S, A}^{2,1}$ denotes the $L_{1}^{2}$ vector valued functions $s=(u, v)$ over the disc satisfying the boundary condition (13). Thus the kernel Ker $\bar{\partial}_{S, A}$, which we will denote by $\mathcal{H}_{S, A}$, consists of holomorphic vector-valued functions $s=(u, v)$ on the disc which satisfy (13) on $\partial D$.

Lemma 5. The operator $\bar{\partial}_{S, A}$ is a real Fredholm operator of index $2 n$.

First, the fact that $\bar{\partial}_{S, A}$ is Fredholm is standard theory, given that the boundary conditions are totally real: i.e., for fixed $\tau \in \partial D$ if two pairs of vectors $(u, v),(i u, i v) \in \mathbf{C}^{n} \times \mathbf{C}^{n}$ satisfy (13) then $u=v=0$. Now to find the index we can deform $(S, A)$ to the constant pair $(0, I)$. The kernel $\mathcal{H}_{0, I}$ consists of holomorphic functions $(u, v)$ with $u=\bar{v}$ over the boundary. It is clear from the Taylor series expansion that the only solution occur when $u, v$ are constant and complex conjugate, so $\mathcal{H}_{0, I}$ has (real) dimension $2 n$. The kernel of the adjoint of $\bar{\partial}_{0, I}$ is given by pairs $(\omega, \theta)$ of holomorphic 1 -forms over the disc with

$$
\overline{\omega(\tau)}=\tau \theta(\tau)
$$

for $\tau \in \partial D$, and one sees again from the Taylor series that there are no non-zero solutions of this equation. Hence the index of $\overline{\partial_{0, I}}$, and so also $\bar{\partial}_{S, A}$, is $2 n$.

As usual, we say that boundary data $(S, A)$ is regular if $\mathcal{H}_{S, A}$ has dimension $2 n$, i.e., if the kernel of the adjoint is zero. 
Definition 1. We say the boundary data $(S, A)$ is super-regular if there are $2 n$ elements $\left(u_{j}, v_{j}\right)$ of $\mathcal{H}_{S, A}$ such that for every $\tau$ in $D$ the vectors $u_{j}(\tau)$ form an $\mathbf{R}$-basis for $\mathbf{C}^{n}$.

Proposition 4. If the boundary data $(S, A)$ is super-regular then it is also regular.

(Notice that this implies that, if $(S, A)$ is super-regular, no non-trivial element of $\mathcal{H}_{S, A}$ can vanish anywhere on the disc.)

To prove Proposition 4 we need a preliminary observation. Given two pairs of vectors $s_{1}=\left(u_{1}, v_{1}\right), s_{2}=\left(u_{2}, v_{2}\right) \in \mathbf{C}^{n} \times \mathbf{C}^{n}$ we write $\Omega\left(s_{1}, s_{2}\right)$ for the standard symplectic form

$$
\Omega\left(s_{1}, s_{2}\right)=u_{1}^{T} v_{2}-u_{2}^{T} v_{2} .
$$

Lemma 6. If $s_{1}, s_{2} \in \mathcal{H}_{S, A}$ then $i \Omega\left(s_{1}(\tau), s_{2}(\tau)\right)$ is real and independent of $\tau$.

To see this observe that the function $i \Omega\left(s_{1}(\tau), s_{2}(\tau)\right.$ is holomorphic in $\tau$ and on the boundary the condition (13) implies that the function takes real values. So the result follows again from the fact that the only such functions are constants.

We now prove Proposition 4. Suppose that there are $2 n$ elements $s_{i}=$ $\left(u_{i}, v_{i}\right)$ of $\mathcal{H}_{S, A}$ as in Definition 1 . We claim that the values $s_{i}(\tau)$ are $\mathbf{C}$ linearly independent at the generic point $\tau$ of the disc. For if not there is a non-trivial linear relation $\sum_{j} \lambda_{j} s_{j}=0$ where the $\lambda_{j}$ are holomorphic functions over the disc. In particular on the boundary we have:

$$
0=\sum \lambda_{j} v_{j}=S\left(\sum_{j} \lambda_{j} u_{j}\right)+A\left(\sum_{j} \lambda_{j} \bar{u}_{j}\right)=A\left(\sum_{j} \lambda_{j} \overline{u_{j}}\right),
$$

and it follows (since $A$ is nondegenerate) that

$$
\sum_{j} \lambda_{j} \bar{u}_{j}=0
$$

This means that, on the boundary

$$
\sum_{j} \bar{\lambda}_{j} u_{j}=\sum_{j} \lambda_{j} u_{j}=0
$$


Thus, if $\lambda_{j}=a_{j}+i b_{j}$ with $a_{j}, b_{j}$ real,

$$
\sum_{j} a_{j} u_{j}=\sum_{j} b_{j} u_{j}=0
$$

and it follows, from the assumption that the $u_{j}$ are $\mathbf{R}$-linearly independent, that $a_{j}=b_{j}=0$. So the holomorphic functions $\lambda_{j}$ vanish on the boundary and hence are identically zero, contrary to the initial assumption.

Suppose now that the space $\mathcal{H}_{S, A}$ has dimension strictly greater than $2 n$ and choose an element $s_{0}$ not in the span of $s_{1}, \ldots s_{2 n}$. By Lemma 6 the symplectic form $\Omega$ defines a skew form on the $2 n+1$-dimensional real vector space $\operatorname{Span}\left(s_{0}, s_{1}, \ldots s_{2 n}\right) \subset \mathcal{H}_{S, A}$. There must be a non-zero element $s$ of Span $\left(s_{0}, s_{1}, \ldots s_{2 n}\right)$ such that

$$
\Omega\left(s, \operatorname{Span}\left(s_{1}, \ldots, s_{2 n}\right)\right)=0 .
$$

But this means that for each $\tau$ in the disc and each $j=1, \ldots, 2 n$,

$$
\Omega\left(s(\tau), s_{j}(\tau)\right)=0 .
$$

But by the fact established above the vectors $s_{1}(\tau), \ldots, s_{2 n}(\tau)$ form a complex basis for $\mathbf{C}^{n} \times \mathbf{C}^{n}$ at the generic point $\tau$ of the disc and at such a point (15) implies that $s(\tau)=0$. Thus $s$ vanishes at the generic point of the disc and so is identically zero. This completes the proof of Proposition 4.

We can now go back to prove Proposition 3. The first thing is to see that that the linearised problem associated to a disc $\psi_{x}$ can be put in the form considered above. To see this in a down-to-earth way we consider first the case when the image of the holomorphic map $f_{x}: D \rightarrow V$ lies in a co-ordinate neighbourhood, with complex co-ordinates $w_{\alpha}$, over which $\omega_{0}$ can be written as $i \bar{\partial} \partial \rho$. Thus the map $f_{x}$ is given in these co-ordinates by its components $f_{x}^{\alpha}(\tau)$. Any nearby map $\tilde{f}: D \rightarrow V$ is likewise represented by its components $\tilde{f}^{\alpha}$. The map $g_{x}: D \rightarrow \mathcal{W}_{V}$ is represented by $f_{x}^{\alpha}$ and $n$ additional fibre components $h^{\beta}(\tau)$, i.e.,

$$
g_{x}(\tau)=\left(f^{\alpha}(\tau), \sum h_{\beta} d \xi_{\beta}\right),
$$

in the canonical co-ordinates on $\mathcal{W}_{V}$ over the co-ordinate patch. Likewise a nearby map $\tilde{g}: D \rightarrow \mathcal{W}_{V}$ is represented by $\left(\tilde{g}^{\alpha}, \tilde{h}^{\alpha}\right)$. The boundary condition - that $\tilde{g}(\tau)$ lies in $\Lambda_{\tau}$-comes down to the equation:

$$
\tilde{h}^{\alpha}(\tau)=\frac{\partial\left(\phi_{\tau}+\rho\right)}{\partial w_{\alpha}}\left(\tilde{f}^{\alpha}(\tau)\right)
$$


for $\tau \in \partial D$. To find the linearised equation we consider a 1-parameter family of such holomorphic map $\left(\tilde{f}_{t}^{\alpha}, \tilde{h}_{t}^{\alpha}\right)$, equal to $\left(f_{x}^{\alpha}, h_{x}^{\alpha}\right)$ when $t=0$, and differentiate (31) with respect to $t$. The resulting equation is

$$
v_{\alpha}(\tau)=\sum_{\beta} \frac{\partial^{2}\left(\phi_{\tau}+\rho\right)}{\partial w_{\alpha} \partial w_{\beta}} u_{\beta}(\tau)+\sum_{\beta} \frac{\partial^{2}\left(\phi_{\tau}+\rho\right)}{\partial w_{\alpha} \partial \bar{w}_{\beta}} \bar{u}_{\beta}(\tau),
$$

where $u_{\alpha}, v_{\alpha}$ are the $t$-derivatives of $\tilde{f}_{t}^{\alpha}, \tilde{h}_{t}^{\alpha}$, evaluated at $t=0$, and the partial derivatives on the right hand side of $(17)$ are evaluated at $f_{x}^{\alpha}(\tau)$. We see then that this is precisely of the form considered above, with

$$
S=\left(\frac{\partial^{2} \phi_{\tau}}{\partial w_{\alpha} \partial w_{\beta}}\right), \quad A=\left(\frac{\partial^{2}\left(\phi_{\tau}+\rho\right)}{\partial w_{\beta} \partial \bar{w}_{\alpha}}\right) .
$$

The global and invariant discussion is much the same. First consider the standard complex symplectic form $\Omega$ on a direct sum $U^{*} \oplus U$, where $U$ is an $n$-dimensional complex vector space and suppose we have a real $2 n$-dimensional subspace $\Lambda \subset U^{*} \oplus U$ which is:

1. Lagrangian with respect to the real part of $\Theta$,

2. symplectic with respect to the imaginary part of $\Theta$,

3. transverse to the summand $U^{*} \subset U^{*} \oplus U$.

Such subspaces are precisely the graphs of $\mathbf{R}$-linear maps

$$
u \mapsto S(u)+A(u)
$$

where $S$ is complex linear and corresponds to a symmetric complex bilinear form on $U$; while $A$ is complex anti-linear and corresponds to a nondegenerate Hermitian form on $U$. The proof of this assertion essentially repeats the discussion of the beginning of Section 2. Now let $\psi_{x}: D \rightarrow D \times \mathcal{W}_{V}$ be one of the maps considered in Proposition 3, of the shape $\psi_{x}(\tau)=\left(\tau, g_{x}(\tau)\right)$. The pull-back of the tangent bundle $\psi_{x}^{*}\left(T\left(D \times \mathcal{W}_{V}\right)\right)$ splits as the direct sum of a trivial bundle $\underline{\mathbf{C}}$ and the pull-back $g_{x}^{*}\left(T \mathcal{W}_{V}\right)$. This latter fits into an exact sequence

$$
0 \rightarrow f_{x}^{*}\left(T^{*} V\right) \rightarrow g_{x}^{*}\left(T \mathcal{W}_{V}\right) \rightarrow f_{x}^{*}(T V) \rightarrow 0
$$

Now all holomorphic bundles over ths disc are trivial so we can trivialise $f_{x}^{*}(T V)$. We may also arrange that this trivialisation is smooth up to the boundary (see the discussion in [3]). Likewise - since all higher cohomology 
groups over the disc vanish - we may split the exact sequence (20), so sections of $g_{x}^{*}\left(T \mathcal{W}_{V}\right)$ are represented by pairs $(u, v)$ of vector valued functions over the disc. We may also choose the trivialisation in such a way that the pull-back of the holomorphic symplectic form $\Theta$ is taken over to the standard form on $\mathbf{C}^{n} \times \mathbf{C}^{n}$. Now, by the preceding discussion, for each $\tau \in \partial D$ the tangent space of $\Lambda_{\tau}$ is represented in this trivialisation by the graph of a linear map of the kind (34). To sum up then, the bundle $E_{\psi_{x}}$ associated to the map $\psi_{x}$ is represented as the trivial holomorphic bundle $\underline{\mathbf{C}}^{n} \oplus \underline{\mathbf{C}^{n}} \oplus \underline{\mathbf{C}}$, so we can represent a section by a triple $(u, v, w)$ say. The boundary conditions for $\tau \in \partial D$ require that

$$
v=S_{\tau} u+A_{\tau} \bar{u}, \operatorname{Im}\left(\tau^{-1} w\right)=0 .
$$

Here $S_{\tau}, A_{\tau}$ are the matrices of the linear maps (19) representing $T \Lambda_{\tau}$ in the chosen trivialisation, and the second equation corresponds to the boundary of the disc in $\mathbf{C}$. There is no coupling between the equations for $(u, v)$ and $w$. The $w$ equation is regular, with index 3 and a solution space of dimension 3 given by the holomorphic vector fields on the disc tangential to the boundary. Thus the map $\psi_{x}$ is regular if and only if the equation for $(u, v)$ is regular in the sense above; in particular this is true if the latter problem is super-regular, by Proposition 4. We say that a holomorphic disc $g_{x}$, with the boundary conditions $\Lambda_{\tau}$ is super-regular if the corresponding linear data $(S, A)$ is super-regular.

To complete the proof of Proposition 3 it only remains to show that any holomorphic disc arising from a solution of Problem 1 is super-regular. But this is rather obvious: we know that there is a $2 n$-dimensional family of holomorphic discs $g_{x}(\tau)=G(\tau, x)$. Thus the partial derivative $\frac{\partial G(\tau, x)}{\partial x}$ defines a linear map $\lambda$ from the tangent space of $V$ at $x$ to the space $\mathcal{H}_{S, A}$ of solutions of our boundary value problem. We also know that for each $\tau \in D$ the map $\gamma_{\tau}$ embeds $V$ as a graph in $\mathcal{W}_{V}$. This means that for non-zero $\xi \in T V_{x}$ the $V$-component of $\lambda(\xi)$ is nowhere vanishing so the images under $\lambda$ of any $\mathbf{R}$-basis for $T V$ give the desired $2 n$ elements of $\mathcal{H}_{S, A}$

\section{Remarks.}

1. There is no real need to use the fact that the sequence (20) can be trivialised holomorphically over the disc, since one can easily adapt the proof of Proposition 4 to general bundles with appropriate structure.

2. The matrix $A_{\tau}$ is just the matrix of the Kahler metric $\omega_{0}+i \bar{\partial} \partial \phi_{\tau}$ on the tangent space of $V$ at $f_{x}(\tau)$, in the chosen trivialisation. 
3. Using the factorisation theorem in finite dimensional loop groups of Chapter 8 of [10] one can always choose a preferred trivialisation of $f_{x}^{*}(T V)$ in which the matrix $A_{\tau}$ is the identity for all $\tau \in \partial D$. The remaining choice is then in the spliting of the exact sequence: changing the splitting allows one to change the $S_{\tau}$ by the boundary value of any holomorphic map from the disc to the symmetric matrices.

\section{Proofs of the main theorems.}

We begin with the proof of Theorem 1: this is just a matter of putting together the work above. Suppose we are given boundary data $F \in$ $C^{\infty}(\partial D \times V)$ for which there exists a smooth solution $\Phi$ to Problem 1 . By Theorem 3, this gives a family of holomorphic discs $g_{x}$, parametrised by $x \in V$, and by Proposition 3 each of these discs is regular. Hence we can apply Proposition 2 to deduce that for any nearby family of submanifolds $\tilde{\Lambda}_{\tau}$ there is a family of holomorphic discs $\tilde{g_{x}}: D \rightarrow \mathcal{W}$ with $\tilde{g_{x}}(\tau) \in \tilde{\Lambda}_{\tau}$ for each $\tau \in \partial D$. In particular if $\tilde{F}$ is close enough to $F$ in $C^{2}$ this is true for the LS-graphs $\tilde{\Lambda}_{\tau}$ defined by $\tilde{F}$. We define $\tilde{G}: D \times V \rightarrow \mathcal{W}$ by $\tilde{G}(\tau, x)=\tilde{g_{x}}(\tau)$, so $\tilde{G}$ is $C^{1}$-close to $G$. Likewise we define $\tilde{\gamma}_{\tau}$ by $\tilde{\gamma}_{\tau}(x)=\tilde{G}(\tau, x)$, and for each $\tau \in D$ the map $\tilde{\gamma}_{\tau}$ is $C^{1}$-close to $\gamma_{\tau}$. In particular we can suppose (assuming that $\tilde{F}$ is sufficiently close to $F$ ) that $\tilde{\gamma}_{\tau}$ is an embedding of $V$ in $\mathcal{W}_{V}$ and the projection $\pi \circ \tilde{\gamma}_{\tau}$ is a diffeomorphism of $V$ (since these are $C^{1}$-open conditions). Then, using Theorem 3 in the other direction, we see that this holomorphic disc-family $\tilde{g}_{x}$ corresponds to a solution to Problem 1 with data $\tilde{F}$.

\subsection{Proof of Theorem 2 .}

We require two Lemmas.

Lemma 7. For any $n \geq 1$ there are matrix-valued maps $S, A$ on $\partial D$ such that the boundary value problem (14) is not super-regular.

Choose holomorphic functions $f, g$ on a neighbourhood of the unit disc $D$ such that $f$ and $g$ have simple zeros at $\tau=1$ and no other zeros on $\partial D$. For example we could take $f(\tau)=g(\tau)=\tau-1$. Then it is easy to verify that the function $\frac{g-\bar{f}}{f}$ extends to a smooth function $\sigma$ on $\partial D$. Thus we have

$$
g=\sigma f+\bar{f}
$$


on $\partial D$. Now let $A_{\tau}$ be the identity matrix for all $\tau \in \partial D$ and let $S_{\tau}$ be the diagonal matrix with diagonal entries $(\sigma, 0, \ldots, 0)$. Then the vector-valued functions

$$
u=(f, 0, \ldots, 0), v=(g, 0, \ldots, 0)
$$

are solutions of the linear system (14) which vanish at the point $\tau=1$. But we have seen that the no solution of a super-regular system can vanish anywhere on the disc, so this system is not super-regular.

Lemma 8. Let $\left(V, \omega_{0}\right)$ be a Kahler manifold and $p$ be a point in $V$. Let $Q_{p}$ denote the set of real quadratic forms on $T V_{p}$. For any $\beta \in Q_{p}$ there is a Kahler metric $\omega_{0}+i \bar{\partial} \partial \phi$ on $V$ such the $\nabla \phi$ vanishes at $p$ and the Hessian of $\phi$ at $p$ is $\beta$. More generally, if $K$ is a compact space and $k \mapsto \beta_{k}$ is a continuous map from $K$ to $Q_{p}$ there is a continuous map $k \mapsto \phi_{k}$ from $K$ to $C^{\infty}(V)$ such that, for each $k \in K, \omega_{0}+i \bar{\partial} \partial \phi_{k}$ is a Kahler metric, $\nabla \phi_{k}$ vanishes at $p$ and the Hessian of $\phi_{k}$ at $p$ is $\beta_{k}$.

Fix a smooth function $f$ on $\mathbf{R}$ such that $f(x)=0$ if $x \geq 2$ and $f(x)=1$ if $x \leq 1$ and for each positive integer $m$ define

$$
f_{m}(x)=f(x)+f\left(\frac{x}{2}\right)+\cdots+f\left(\frac{x}{2^{m}}\right) .
$$

Then it is clear that $x f_{m}^{\prime}(x), x^{2} f_{m}^{\prime \prime}(x)$ are bounded, independent of $m$ and $x$. If we define $g_{m}$ by

$$
g_{m}(x)=\frac{1}{m+1} f_{m}\left(2^{m+1} x\right)
$$

then $g_{m}(x)=1$ for small positive $x$, while $g_{m}(x)$ vanishes if $x>1$ and $\left|x g_{m}^{\prime}(x)\right|,\left|x^{2} g_{m}^{\prime \prime}(x)\right|$ are bounded by $C / m$ for some fixed $C$.

Now choose local complex co-ordinates $z_{\alpha}$ around the point $p$ in $V$, without loss of generality defined on the unit ball in $\mathbf{C}^{n}$. In these co-ordinates a quadratic form $\beta \in Q_{p}$ can be regarded as a quadratic function on this co-ordinate ball and, for suitably large $m$, we define

$$
\phi_{\beta}(z)=g_{m}(|z|) \beta(z),
$$

extended by zero over the rest of $V$. This has the correct Hessian, since $g_{m}(x)=1$ for small $x$, and

$$
\left|\bar{\partial} \partial \phi_{\beta}\right| \leq C^{\prime}\left(|z|^{2}\left|g_{m}^{\prime \prime}(|z|)\right|+|z|\left|g_{m}^{\prime}(|z|)\right|\right)=O\left(m^{-1}\right) .
$$

Thus $\omega_{0}+i \bar{\partial} \partial \phi_{\beta}$ is positive if $m$ is large enough. The extension of the argument to families is immediate. 
We now return to the proof of Theorem 2. We fix boundary data $S_{\tau}, A_{\tau}$ of a linear system which is not super-regular, as in Lemma 7, and a point $p$ in $V$. In a basis of $T V_{p}$ the data $S_{\tau}, A_{\tau}$ can be regarded as defining an element of $Q_{p}$, for each $\tau \in \partial D$. By Lemma 8 we can find a family of Kahler potentials $\phi_{\tau}$ such that $\nabla \phi_{\tau}$ vanishes at $p$ and the Hessian at that point corresponds to $\left(S_{\tau}, A_{\tau}\right)$ via (18). The condition on the first derivative means that the LS-graphs $\Lambda_{\tau}$ defined by the $\phi_{\tau}$ all pass though a fixed point $\xi$ in $\mathcal{W}$. Thus the constant map $g: D \rightarrow \mathcal{W}$, mapping the whole disc to $\xi$, is a solution of the boundary value problem defined by the $\Lambda_{\tau}$ and, by construction, this holomorphic disc is not super-regular (for these boundary conditions). So if we let $F(x, \tau)=\phi_{\tau}(x)$ and if there is a smooth solution to Problem 1 for this boundary data $F$ the holomorphic disc $g$ cannot appear as one of the family associated to the solution. Now choose a two-parameter family $\phi_{\tau, t}$ of Kahler potentials on $V$, for $t \in[0,1]$, such that $\phi_{\tau, 1}=\phi_{\tau}$ and $\phi_{\tau, 0}=0$ for all $\tau \in \partial D$, and such that the derivative $\nabla \phi_{\tau, t}$ vanishes at $p$ for all $\tau, t$. Then for each $t \in[0,1]$ the constant holomorphic disc $g$ satisfies the boundary conditions asociated to $\phi_{\tau, t}$. Suppose that a solution to Problem 1 exists for the boundary data $\phi_{\tau, t}$, for each $t \in[0,1]$. Let $U \subset[0,1]$ be the set of parameters $t$ such that the disc $g$ appears in the family of holomorphic discs associated to the solution. Thus the point $t=0$ certainly lies in $U$ (all the discs in the family are constant in that case), but we have shown that $t=1$ does not lie in $U$. On the other hand Proposition 2 implies that $U \subset[0,1]$ is both open and closed, which gives a contradiction.

\section{References.}

[1] E. Bishop, Differentiable manifolds in complex Euclidean space, Duke Math. Jour., 32 (1965), 1-22.

[2] X-X. Chen, The space of Kahler metrics, Journal of Differential Geometry, 20 (2000), 189-234.

[3] S.K. Donaldson, Boundary value problems for Yang-Mills fields, Jour. Geometry and Physics, 8 (1992), 89-122.

[4] S.K. Donaldson, Remarks on gauge theory, complex geometry and 4manifold topology, in 'Fields Medalists Lectures (M. Atiyah and D. Iagonalitzer eds.)', World Scientific, (1997), 384-403. 
[5] S.K. Donaldson, Symmetric spaces, Kahler geometry and Hamiltonian dynamics, in 'Northern California Symplectic Geometry Seminar', AMS Translations, 196 (1999), 13-34.

[6] S.K. Donaldson, Moment maps and diffeomorphisms, Asian Journal of Maths., 3 (1999), 1-15.

[7] M. Gromov, Pseudo-holomorphic curves in symplectic manifolds, Inventiones Math., 82 (1985), 307-347.

[8] T. Mabuchi, Some symplectic geometry on compact kahler manifolds, Osaka Jour. Math., 24 (1987), 227-252.

[9] R. Moriyon, Regularity of the Dirichlet Problem for the degenerate complex Monge-Ampere equation, Commun. Pure and Applied Mathematics, 35 (1982), 1-27.

[10] A. Pressley and G. Segal, Loop Groups, Oxford U.P., 1986.

Imperial College, LoNdoN 